\title{
Direct Observation of Charge Order and an Orbital Glass State in Multiferroic $\mathrm{LuFe}_{2} \mathrm{O}_{4}$
}

\author{
A. M. Mulders, ${ }^{1,2}$ S. M. Lawrence, ${ }^{1}$ U. Staub,${ }^{3}$ M. Garcia-Fernandez, ${ }^{3}$ V. Scagnoli, ${ }^{4}$ C. Mazzoli, ${ }^{4}$ E. Pomjakushina, ${ }^{5,6}$ \\ K. Conder, ${ }^{5}$ and Y. Wang ${ }^{7}$ \\ ${ }^{1}$ Department of Imaging and Applied Physics, Curtin University of Technology, Perth, WA 6845, Australia \\ ${ }^{2}$ The Bragg Institute, Australian Nuclear Science and Technology Organisation, Lucas Heights, NSW 2234, Australia \\ ${ }^{3}$ Swiss Light Source, Paul Scherrer Institut, 5232 Villigen PSI, Switzerland \\ ${ }^{4}$ European Synchrotron Radiation Facility, BP 220, 38043 Grenoble Cedex 9, France \\ ${ }^{5}$ Laboratory for Developments and Methods, Paul Scherrer Institut, 5232 Villigen PSI, Switzerland \\ ${ }^{6}$ Laboratory for Neutron Scattering, ETHZ \& PSI, 5232 Villigen, Switzerland \\ ${ }^{7}$ Department of Applied Physics, The Hong Kong Polytechnic University, Hong Kong, China
}

(Received 15 December 2008; published 12 August 2009)

\begin{abstract}
Geometrical frustration of the Fe ions in $\mathrm{LuFe}_{2} \mathrm{O}_{4}$ leads to intricate charge and magnetic order and a strong magnetoelectric coupling. Using resonant $\mathrm{x}$-ray diffraction at the $\mathrm{Fe} K$ edge, the anomalous scattering factors of both Fe sites are deduced from the $(h / 3 k / 3 l / 2)$ reflections. The chemical shift between the two types of $\mathrm{Fe}$ ions equals $4.0(1) \mathrm{eV}$ corresponding to full charge separation into $\mathrm{Fe}^{2+}$ and $\mathrm{Fe}^{3+}$. The polarization and azimuthal angle dependence of the superlattice reflections demonstrate the absence of differences in anisotropic scattering revealing random orientations of the $\mathrm{Fe}^{2+}$ orbitals characteristic of an orbital glass state.
\end{abstract}

DOI: 10.1103/PhysRevLett.103.077602

PACS numbers: 77.80.-e, 61.05.C-, 75.80.+q

New materials that exhibit strong magnetoelectric coupling are fascinating because a large coupling between ferroelectric and magnetic interactions is rare, and its origin often unclear. Competing interactions lead to novel ground states that give rise to unusual material properties, i.e., the coexistence of spontaneous magnetic and ferroelectric order [1]. The ability to control electric polarization with a magnetic field or the magnetization with an electric field [2-4] makes these multiferroic materials promising candidates for novel applications. In most ferroelectric materials, electric polarization arises from covalent bonding between anions and cations or the orbital hybridization of electrons. Alternatively ferroelectric polarization may arise from frustrated charge order as reported for $\mathrm{LuFe}_{2} \mathrm{O}_{4}$ [5]. This compound is of particular interest because, in addition to ferroelectricity, magnetism originates from the same Fe ions and this holds the promise of strong magnetoelectric coupling. The ferroelectric and magnetic order take place at and near ambient temperature which provides the potential for room temperature multiferroics.

The crystal structure of $\mathrm{LuFe}_{2} \mathrm{O}_{4}$ consists of a triangular double layer of iron ions, forming trigonal bipyramids with five oxygen nearest neighbors, in which an equal amount of $\mathrm{Fe}^{2+}$ and $\mathrm{Fe}^{3+}$ are believed to coexist at the same site [6]. The occurrence of different charge order schemes has already been studied in more detail theoretically and experimentally $[7,8]$. $\mathrm{LuFe}_{2} \mathrm{O}_{4}$ adopts a ferroelectric ground state below $\sim 350 \mathrm{~K}$, while below $\sim 250 \mathrm{~K}$ two-dimensional magnetic order is established in the triangular planes which enhances the ferroelectric polarization by $20 \%$, illustrating coupling between the magnetic and ferroelectric order [5,9]. The observation of the $(1 / 31 / 313 / 2)$ reflection using resonant $\mathrm{x}$-ray Bragg diffraction (RXD) further supports the existence of charge ordering [10].

RXD has become a powerful technique to study charge, orbital, and magnetic arrangements. Tuning the energy of the incoming radiation to an absorption edge permits recording Bragg reflections with enhanced sensitivity to the specific ion and its electronic configuration. In the case of the $\mathrm{Fe} K$ edge, the incident $\mathrm{x}$ rays virtually excite an electron from the $1 s$ core level to the empty $4 p$ states, followed by a decay of the electron back to the core hole. This effect results in a significant variation in the atomic scattering factors of the $\mathrm{Fe}$ ions for $\mathrm{x}$-ray energies close to the $\mathrm{Fe} K$ edge. The atomic scattering factors are also affected by variations in charge state, ordered aspherical electron densities, or ordered magnetic moments. In particular, asphericity of the atomic electron density results in anisotropy of the tensor of x-ray susceptibility (ATS). Each of these phenomena has a specific dependence on the polarization of the incoming radiation and the orientation of the sample with respect to the scattering geometry. The significance of RXD has been demonstrated, among others, in the manganites [11], nickelates [12], and magnetite [13].

In a perfectly charge ordered state, each site may be considered as having an excess and a deficiency of half an electron respectively, compared to the average ion valence of $\mathrm{Fe}^{2.5+}$. Alternatively, charge disproportionation with fractional charges may exist as exemplified in nickelates $[14,15]$. Frustration arises because every excess charge prefers a deficiency charge as a neighbor which is not possible on a triangular lattice. However, in the presence of a second triangular layer a net transfer of charge from the first layer to second occurs because then it is possible to 
have charge order on each layer in a honeycomb lattice arrangement $[16,17]$.

The crystal field of the trigonal bipyramids splits the $3 d$ states of $\mathrm{LuFe}_{2} \mathrm{O}_{4}$ into two doublets $\left(d_{x y} / d_{x^{2}-y^{2}}\right.$ and $\left.d_{x z} / d_{y z}\right)$ and a singlet $\left(d_{z^{2}}\right)$ [18]. $\mathrm{Fe}^{3+}$ with five $3 d$ electrons is spherical while $\mathrm{Fe}^{2+}$ with six $3 d$ electrons exhibits doubly degenerate orbital degree of freedom in the $d_{x y} / d_{x^{2}-y^{2}}$ ground state.

We present RXD data with azimuthal angle and polarization analysis, and our results clarify that the charge order is close to electronic states of $\mathrm{Fe}^{2+}$ and $\mathrm{Fe}^{3+}$, in contrast to small values of disproportionation observed in nickelates [15] and manganites [11]. Moreover, the absence of scattering due to ATS demonstrates a glass state of the $\mathrm{Fe}^{2+}$ orbitals in agreement with calculations [18].

Polycrystalline $\mathrm{LuFe}_{2} \mathrm{O}_{4}$ was prepared by a solid state reaction as reported in Ref. [19]. Starting materials of $\mathrm{Lu}_{2} \mathrm{O}_{3}$ and $\mathrm{Fe}_{2} \mathrm{O}_{3}$ with $99.99 \%$ purity were mixed, pressed into pellets, and sintered at $1200{ }^{\circ} \mathrm{C}$ during $6 \mathrm{~h}$ in $\mathrm{H}_{2} / \mathrm{He} / \mathrm{CO}_{2}$ atmosphere $\left(\mathrm{H}_{2} / \mathrm{CO}_{2}\right.$ ratio $\left.1 / 3\right)$ and quenched into ice water. After grinding, the obtained powder was hydrostatically pressed and sintered at the same conditions during $3 \mathrm{~h}$. The crystal growth was carried out using an optical floating zone furnace with four $1000 \mathrm{~W}$ halogen lamps as a heat source, growth rate $1 \mathrm{~mm} / \mathrm{h}, 2$ bar pressure of $\mathrm{CO}_{2} / \mathrm{CO}$ mixture ( $5 / 2$ ratio). The single crystal was cut and samples have been polished perpendicular to the [001] and [110] directions. The magnetic ordering temperature was determined with a SQUID magnetometer at $240 \mathrm{~K}$, and pyroelectric current measurement confirmed ferroelectric order below $\sim 330 \mathrm{~K}$ and enhanced ferroelectric order below $\sim 220 \mathrm{~K}$.

Various $(h / 3 \mathrm{k} / 3 \mathrm{l} / \mathrm{2})$ superlattice reflections were recorded at the Fe $K$ edge at beam line ID20 of the ESRF [20]. Polarization analysis was performed using a $\mathrm{MgO}(2$ 22 ) analyzer crystal of which the polarization efficiency was determined at 0.98 for the energy of the Fe $K$ edge. In addition, RXD was recorded at the MS beam line at the SLS (see also Ref. [21]) using the Pilatus 2D detector [22]. The background, mainly originating from the fluorescence of the sample, was determined from selected border regions of the area detector and subtracted. The integrated diffracted intensity was corrected for polarization, absorption, and sample geometry. The absorption, $\mu(E)$, was obtained from the (006) reflection by iteration of the calculated anomalous intensity according to space group $R \overline{3} m$ and $\mu(E)$ deduced from the ratio between calculated and integrated intensity recorded with the $2 \mathrm{D}$ detector. In addition, $\mu(E)$ was recorded at the X-ray Absorption Spectroscopy beam line at the Australian Synchrotron using powdered $\mathrm{LuFe}_{2} \mathrm{O}_{4}$ pressed with cellulose and $\mathrm{Fe}$ foil as energy calibration. It confirmed the validity of the method to obtain $\mu(E)$ from the (006). RXD recorded at the SLS was used to analyze the energy dependence while RXD recorded at ID20 was used to investigate polarization dependence and rotation about the scattering vector $\mathbf{q}$ (azimuthal angle $\Psi$ ). $\Psi$ is defined zero when [110] and [001] are in the scattering plane.

The $(h / 3 k / 37 / 2)$ reflections show identical RXD [21] while the magnitude and sign of the RXD observed at the $(2 / 3-1 / 3 l / 2)$ reflections depends on $l$ (Fig. 1). The relative magnitude and shape are constant between 10 and $300 \mathrm{~K}$ and the diffracted intensity gradually disappears above the ferroelectric ordering temperature as illustrated in the inset of Fig. 1. Thomson scattering associated with the crystallographic distortion that accompanies the ferroelectric polarization dominates the diffracted intensity before and after the edge. The variation of RXD with $l$ suggests that the scattering amplitude related to the anomalous diffraction of the Fe ions adds phase shifted contributions to the scattering amplitude from the structural distortion, depending on the $l$ index. The structure factor is $F=\sum_{j} f_{j} \exp \left(i \mathbf{q} \cdot \mathbf{r}_{j}\right)$ with $f_{j}$ the atomic form factor of atom $j$ and $\mathbf{r}_{j}$ its position in the unit cell. To analyze the energy dependent intensity we separate $F$ into an energy independent and an energy dependent term, $F=$ $F_{\mathrm{Fe}, \mathrm{Lu}, \mathrm{O}}^{0}+F_{\mathrm{Fe}}(E)$, where the first term is the Thomson scattering of $\mathrm{Fe}, \mathrm{Lu}$, and/or $\mathrm{O}$ ions and the second term is anomalous diffraction due to the $\mathrm{Fe}$ ions. These terms are written as $F_{\mathrm{Fe}, \mathrm{Lu}, \mathrm{O}}^{0}=A+i B$ and $F_{\mathrm{Fe}}(E)=\sum_{j}\left[f_{j}^{\prime}(E)+\right.$ $\left.i f_{j}^{\prime \prime}(E)\right] e^{i q \cdot r_{j}}$ where $A$ and $B$ are the real and imaginary components of the nonresonant structure factor and $f_{j}^{\prime}(E)$ and $f_{j}^{\prime \prime}(E)$ are the real and imaginary components of the

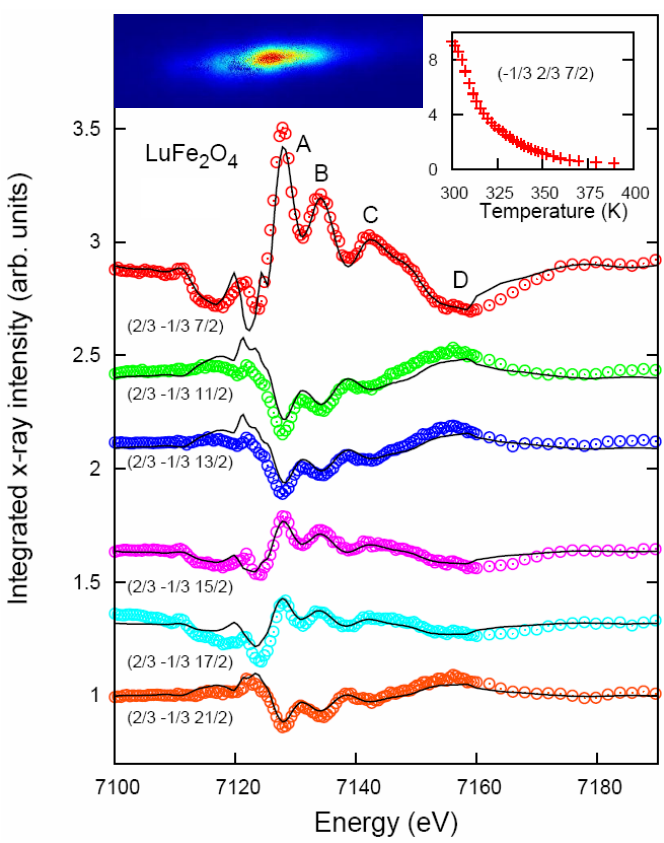

FIG. 1 (color online). Integrated resonant diffraction intensity of the charge order $(2 / 3-1 / 3 l / 2)$ reflections recorded with 2D detector at $T=15 \mathrm{~K}$ (see inset top left for $l=11$ ), corrected for background, polarization, absorption and sample geometry, normalized (shifted for clarity) and compared to charge order model described in the text. The inset (top right) shows that the superlattice intensity gradually disappears at the ferroelectric ordering temperature. For $A, B, C$, and $D$, see text. 
anomalous scattering factor of the Fe ions. $f_{j}^{\prime}(E)$ and $f_{j}^{\prime \prime}(E)$ are related through the Kramers-Kronig relation.

For the superlattice reflections of this study $F_{\mathrm{Fe}}(E)$ almost cancels except for the fact that Fe ions in different local electronic environments exhibit different energies of the $1 s$ and the $4 p$ states. This results in different transition energies; for example, a chemical shift of $4.5 \mathrm{eV}$ has been reported for $f_{j}^{\prime \prime}(E)$ between $\mathrm{Fe}^{2+}$ and $\mathrm{Fe}^{3+}$ in water complexes [23]. In addition, ATS arises when the $4 p$ states are split. The extended $4 p$ orbitals are sensitive to local distortions, and orbital order of the $3 d$ shell gives rise to splitting of the $4 p$ states, for example, via the associated Jahn-Teller effect.

Differences in ATS give rise to a modulation in RXD intensity as function of azimuthal angle that is related to the symmetry of the distortion. Combined with the Thomson scattering, whose diffracted intensity is independent of azimuthal angle, this results in a change of the relative amplitude. Figure 2(a) shows this is not observed in $\mathrm{LuFe}_{2} \mathrm{O}_{4}$ in contrast to orbitally ordered manganites [11]. Furthermore, a significant contribution in diffraction with rotated polarization is expected for ATS, whereas polarization analysis shows that the diffracted intensity with rotated polarization, $\sigma \pi^{\prime}$, is weak and accounted for by the unrotated $\sigma \sigma^{\prime}$ contribution within the polarization resolution of the analyzer [see Fig. 2(b)]. The $(1 / 31 / 3 l / 2)$ reflections of Fig. 2 have arbitrary angles $\phi_{l}$ with the [001] direction $\left(\phi_{3}=73^{\circ}\right.$ and $\left.\phi_{31}=18^{\circ}\right)$ so that ATS is not canceled to zero by symmetry if there is orbital order. Moreover, polarization analysis of various other $(1 / 31 / 3 l / 2)$ reflections and several azimuthal angles recorded for $l=29$ yielded the same result [21].

Both aspects signal the absence of local asymmetric distortions associated with orbital order and point to a rather symmetrical expansion or contraction of the trigonal

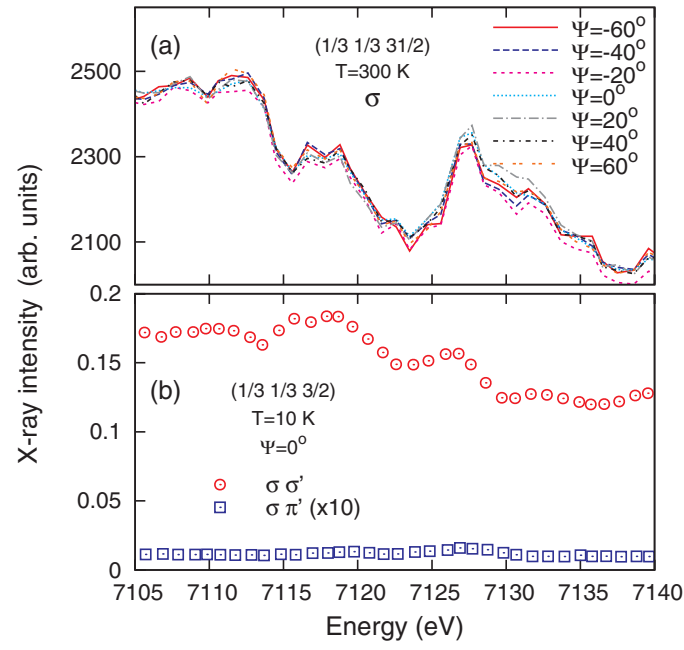

FIG. 2 (color online). (a) Intensity of the $(1 / 31 / 331 / 2)$ reflection as function of azimuthal angle $\Psi$. (b) Intensity of the $(1 / 31 / 33 / 2)$ reflection recorded with $\sigma \sigma^{\prime}$ and $\sigma \pi^{\prime}$ radiation. The latter intensity is multiplied by 10 for clarity. bipyramids in the $a b$ plane, changing the electronic density at the resonant ion while the symmetry of the scattering factor is unaltered.

The energy dependencies of the $(h / 3 \mathrm{k} / 3 \mathrm{l} / 2)$ reflections are examined with two different charge states of the Fe ions, labeled 1 and 2 , and the structure factor at $(h / 3 k / 3 l / 2)$ equals

$$
F_{l}(E) \propto A_{l}^{\prime}+i B_{l}^{\prime}+f_{1}^{\prime}(E)+i f_{1}^{\prime \prime}(E)-f_{2}^{\prime}(E)-i f_{2}^{\prime \prime}(E),
$$

where $A_{l}^{\prime}$ and $B_{l}^{\prime}$ are real constants of arbitrary magnitude. In particular, $B_{l}^{\prime}$ is nonzero due to the charge order breaking inversion symmetry. The sum $\left(f_{1}^{\prime \prime}+f_{2}^{\prime \prime}\right)$ is obtained from $\mu(E)$ using the optical theorem. In this work we aim to deduce $f_{1}(E)$ and $f_{2}(E)$ from the RXD spectra without any assumptions on the local distortion. To test the robust ness of the specific energy dependencies, the series of $(2 / 3-1 / 3 l / 2)$ reflections are refined with different methods. Besides fully independent $f_{1}(E)$ and $f_{2}(E)$, we added the constraint $f_{2}\left(E-\frac{1}{2} \Delta\right)=f_{1}\left(E+\frac{1}{2} \Delta\right)$, where $\Delta$ equals the chemical shift. Moreover, we have calculated $f^{\prime \prime}(E)$ with FDMNES using the muffin-tin approximation [21,24] in $R \overline{3} m$ and used $f_{2}\left(E-\frac{1}{2} \Delta\right)=f_{1}\left(E+\frac{1}{2} \Delta\right)$ to fit the RXD data. The chemical shift between $f_{1}^{\prime \prime}(E)$ and $f_{2}^{\prime \prime}(E)$ is similar in the three refinements and equals $4.0(1) \mathrm{eV}$. The first refinement resulted in distinct energy dependencies for $f_{1}(E)$ and $f_{2}(E)$. This is understood as a result of the experimental uncertainty of the RXD spectra and minimizing $f_{2}\left(E-\frac{1}{2} \Delta\right)-f_{1}\left(E+\frac{1}{2} \Delta\right)$ was added in a further refinement to promote similarity between $f_{1}(E)$ and $f_{2}(E)$. Figure 1 illustrates the resulting fits and $f_{1}^{\prime \prime}(E)$ and $f_{2}^{\prime \prime}(E)$ are presented in Fig. 3. Both $f_{1}^{\prime \prime}(E)$ and $f_{2}^{\prime \prime}(E)$ exhibit characteristic features at similar energies above the edge; however, their magnitudes are different. Figure 4 compares the three different models for the $(2 / 3-1 / 37 / 2)$ reflection.

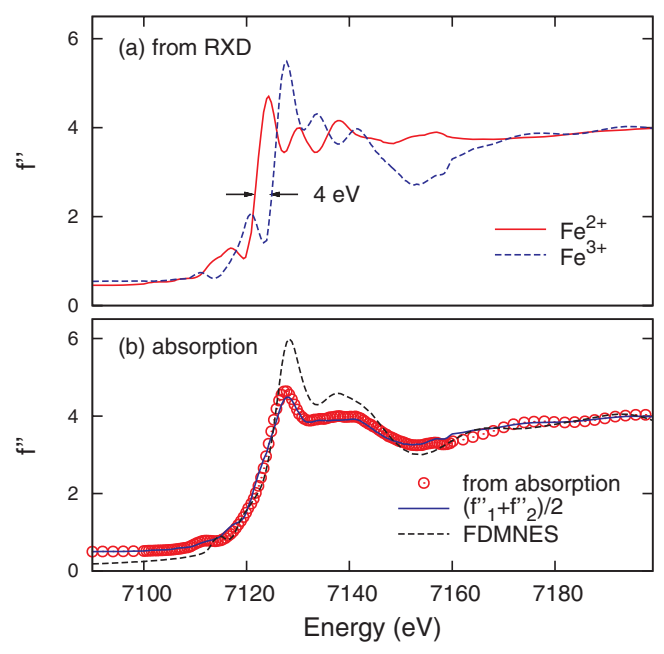

FIG. 3 (color online). (a) $f_{1}^{\prime \prime}(E)$ and $f_{2}^{\prime \prime}(E)$ deduced from the refinement of RXD (Fig. 1) via the method described in the text and (b) its average compared with FDMNES calculation in $R \overline{3} \mathrm{~m}$ (dashed curve) and $\left[f_{1}^{\prime \prime}(E)+f_{2}^{\prime \prime}(E)\right] / 2$ from $\mu(E)$ (open circles). 


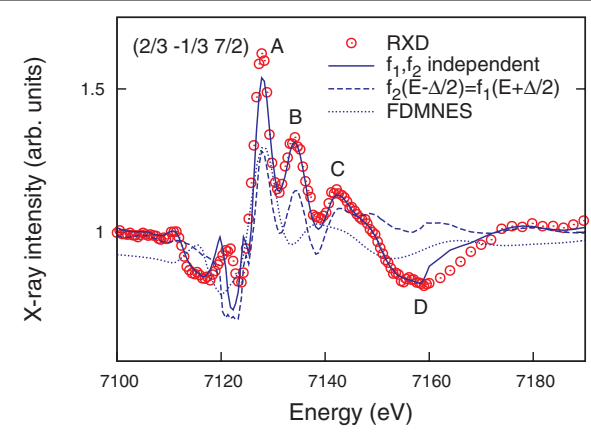

FIG. 4 (color online). RXD compared to three models of charge order as described in the text. The solid curve corresponds to $f_{1,2}^{\prime \prime}(E)$ as presented in Fig. 3. The dashed curve and the dotted curve correspond to identical $f_{1}(E)$ and $f_{2}(E)$ except for a chemical shift. The dashed curve is fitted to the RXD data whereas the dotted curve corresponds to $f_{1,2}(E)$ calculated with FDMNES.

The chemical shift between $f_{1}^{\prime \prime}(E)$ and $f_{2}^{\prime \prime}(E)$ of 4.0 (1) $\mathrm{eV}$ corresponds to the chemical shift between $\mathrm{Fe}^{2+}$ and $\mathrm{Fe}^{3+}$ in $\mathrm{FeO}$ and $\mathrm{Fe}_{2} \mathrm{O}_{3}$ and confirms $\mathrm{Fe}^{2+} / \mathrm{Fe}^{3+}$ charge order. Besides the invariable chemical shift there are dissimilarities between the models. The double feature indicated with $B$ and $C$ in Fig. 4 is not reproduced by the fit based on the FDMNES calculations. In addition, the broad feature, labeled $D$, cannot be accounted for with $f_{2}(E-$ $\left.\frac{1}{2} \Delta\right)=f_{1}\left(E+\frac{1}{2} \Delta\right)$. Yet, features $B, C$, and $D$ are either in or out of phase with the Thomson scattering (see Fig. 1). This signifies that the $\mathrm{Fe}-\mathrm{O}$ bonds are distinct at each $\mathrm{Fe}$ site.

The long-range order of the $\mathrm{Fe}^{2+}$ orbitals leads to ATS that is distinct from that of the spherical $\mathrm{Fe}^{3+}$ ion. Such difference in ATS between the $\mathrm{Fe}^{2+}$ and $\mathrm{Fe}^{3+}$ ions is absent and evidence of random orientations of the $\mathrm{Fe}^{2+}$ orbitals. Our findings are consistent with the unconventional orbital state calculated by Nagano et al. with frustrated orbital orientations and large degeneracy in the ground state [18].

The orbital state critically influences the superexchange interaction and is essential to understand the magnetoelectric properties of $\mathrm{LuFe}_{2} \mathrm{O}_{4}$. In contrast to the manganites, where the orbital liquid state is discussed to be associated with the ferromagnetic and metallic state [25], $\mathrm{LuFe}_{2} \mathrm{O}_{4}$ shows orbital disorder in the ferroelectric state. As such it is more appropriate to classify $\mathrm{LuFe}_{2} \mathrm{O}_{4}$ as an orbital glass, adding further frustration to the already frustrated magnetic interactions of Ising spins on a triangular lattice.

$\mathrm{Fe}_{3} \mathrm{O}_{4}$ and $\mathrm{LuFe}_{2} \mathrm{O}_{4}$ both exhibit ferrimagnetic order combined with magnetoelectric effects [26], but their charge and orbital states are evidently distinct. $\mathrm{Fe}_{3} \mathrm{O}_{4}$ shows merely fractional charge order [13], and recently order of the $t_{2 g}$ orbitals was observed below the Verwey transition [27]. While the superexchange is prevailing in $\mathrm{Fe}_{3} \mathrm{O}_{4}$, Coulomb interactions dominate in $\mathrm{LuFe}_{2} \mathrm{O}_{4}$, resulting in charge order and crystallographic distortions that do not accommodate orbital alignment. The reduced amplitude of $f^{\prime \prime}$ of $\mathrm{Fe}^{2+}$ just above the edge [see Fig. 3(a)] is possibly indicative of this frustration and the subject of further investigation.

Finally it is noted that cooling from $380 \mathrm{~K}$ in an electric field of $\sim 1 \mathrm{MV} / \mathrm{m}$ did not result in a significant change of the $(h / 3 k / 3 l / 2)$ superlattice reflections which contradicts the suggestion that ferroelectric charge order is the ground state only after electric field cooling [28].

In conclusion, our RXD data show an almost complete $\mathrm{Fe}^{2+} / \mathrm{Fe}^{3+}$ charge order as the origin of the superlattice in $\mathrm{LuFe}_{2} \mathrm{O}_{4}$. The $\mathrm{Fe}$ scattering factors are isotropic and in agreement with frustrated and random orientations of the $\mathrm{Fe}^{2+}$ orbitals, forming an orbital glass state.

We thank the beam line staff of X04SA and, in particular, Phil Wilmot and Dominique Meister for its excellent support, Chris Glover and the Australian Synchrotron for XANES measurement, Alec Duncan and the CMST for the use of MATLAB routines, and acknowledge the ESRF for provision of beam time. This work was partly performed at the SLS of the Paul Scherrer Institut, Villigen, Switzerland. We acknowledge financial support from the NCCR MaNEP, the Swiss National Science Foundation, AINSE, and Access to Major Research Facilities Programme.

[1] T. Kimura et al., Nature (London) 426, 55 (2003).

[2] T. Zhao et al., Nature Mater. 5, 823 (2006).

[3] Y. Yamasaki et al., Phys. Rev. Lett. 98, 147204 (2007).

[4] Y. Bodenthin et al., Phys. Rev. Lett. 100, 027201 (2008).

[5] N. Ikeda et al., Nature (London) 436, 1136 (2005).

[6] T. Sekine and T. Katsura, J. Solid State Chem. 17, 49 (1976).

[7] H. J. Xiang and M.-H. Whangbo, Phys. Rev. Lett. 98, 246403 (2007).

[8] Y. Zhang et al., Phys. Rev. Lett. 98, 247602 (2007).

[9] J. Iida et al., J. Phys. Soc. Jpn. 62, 1723 (1993).

[10] N. Ikeda, J. Phys. Condens. Matter 20, 434218 (2008).

[11] J. Herrero-Martin et al., Phys. Rev. B 70, 024408 (2004).

[12] V. Scagnoli et al., Phys. Rev. B 72, 155111 (2005).

[13] E. Nazarenko et al., Phys. Rev. Lett. 97, 056403 (2006).

[14] J. Alonso et al., Phys. Rev. Lett. 82, 3871 (1999).

[15] U. Staub et al., Phys. Rev. Lett. 88, 126402 (2002).

[16] G. H. Wannier, Phys. Rev. 79, 357 (1950).

[17] A. Nasu et al., Phys. Rev. B 78, 024416 (2008).

[18] A. Nagano et al., Phys. Rev. Lett. 99, 217202 (2007).

[19] J. Iida, S. Takekawa, and N. Kimizuka, J. Cryst. Growth 102, 398 (1990).

[20] L. Paolasini, J. Synchrotron Radiat. 14, 301 (2007).

[21] See EPAPS Document No. E-PRLTAO-103-023934 for more details. For more information on EPAPS, see http:// www.aip.org/pubservs/epaps.html.

[22] C. Broennimann et al., J. Synchrotron Radiat. 13, 120 (2006).

[23] M. Benfatto et al., Chem. Phys. 282, 441 (2002).

[24] Y. Joly, Phys. Rev. B 63, 125120 (2001).

[25] Y. Tokura and N. Nagaosa, Science 288, 462 (2000).

[26] D. I. Khomskii, J. Magn. Magn. Mater. 306, 1 (2006).

[27] J. Schlappa et al., Phys. Rev. Lett. 100, 026406 (2008).

[28] M. Angst et al., Phys. Rev. Lett. 101, 227601 (2008). 